\title{
Carcinoid tumor and papillary thyroid carcinoma arising from ovarian mature cystic teratoma: two cases of an extraordinary malignant transformation
}

\author{
Jimmy A. Billod*, Joella G. Saure, Katrina Mae A. Natavio
}

Department of Obstetrics and Gynecology, Baguio General Hospital and Medical Center, Baguio City, Philippines

Received: 24 January 2021

Accepted: 02 March 2021

\author{
*Correspondence: \\ Dr. Jimmy A. Billod, \\ E-mail: jabillodmd@gmail.com
}

Copyright: () the author(s), publisher and licensee Medip Academy. This is an open-access article distributed under the terms of the Creative Commons Attribution Non-Commercial License, which permits unrestricted non-commercial use, distribution, and reproduction in any medium, provided the original work is properly cited.

\begin{abstract}
The most common malignant transformation of mature cystic teratoma of the ovary (MCTO) is squamous cell carcinoma, whereas, carcinoid tumor and papillary thyroid carcinoma are extremely extraordinary. This paper presents two rare cases of malignant transformation from MCTO. First, in a 33 year old, nulligravid, who underwent oophorocystectomy for a dermoid cyst. Histopathologic and Immunohistochemical studies revealed a carcinoid tumor arising from a mature cystic teratoma. Second, in a 52 year old who underwent, total abdominal hysterectomy with bilateral salpingo-oophorectomy for an ovarian new growth, with a final histopathologic and immunohistochemical analysis showed follicular variant of papillary carcinoma arising from mature cystic teratoma. Preoperative diagnosis of MCTO can be made through physical examination and imaging however not the concealed malignancy. Thorough extensive sampling and histopathologic examination of a dermoid cyst is necessary to detect malignant transformation. The optimal management of the disease is uncertain because of its rarity, but complete excision of tumor renders excellent prognosis.
\end{abstract}

Keywords: Carcinoid tumor, Malignant transformation of teratomas, Mature cystic teratoma, Papillary thyroid carcinoma

\section{INTRODUCTION}

Mature cystic teratoma (MCT) is the most common germ cell tumor of ovary, comprising $10-25 \%$ of all ovarian neoplasms. It is generally benign, but malignant transformation of mature cystic teratoma of the ovary (MCTO) is reported as a rare phenomenon occurring in 1$3 \%$ of all MCTs, usually in postmenopausal women. ${ }^{1}$

The most common malignant tumor in MCT is squamous cell carcinoma, whereas, carcinoid tumor and papillary thyroid carcinoma are extremely rare. Other tumors arising in MCT include adenocarcinoma, melanoma, transitional cell carcinoma, and sarcoma. Malignant transformation is rarely diagnosed preoperatively but only postoperative pathological examination. ${ }^{2}$ No clinical, radiological, and biological signs are specific to malignant transformation of
MCT. ${ }^{3}$ Between January 2016 and December 2019, the prevalence rate of malignant transformation in MCTO in our institution is $6 \%$, commonly squamous cell carcinoma (SCCA). This paper aims to report two unique cases of malignant transformation, a carcinoid tumor and a follicular variant of papillary thyroid carcinoma arising from MCTO and deliver a review in the light of recent literature.

\section{CASE REPORT}

\section{Case 1}

A 33 year-old, nulligravid who presented with a 2 year history a gradually enlarging left lower quadrant mass with no associated signs and symptoms. One month prior to admission, there was increase in the size of the mass for 
which she consulted. Pelvic ultrasound showed normal sized anteverted uterus measuring $5.5 \times 3.9 \times 4.4 \mathrm{~cm}$ with homogenous myometrial echotexture (Figure 1a). There is a complex mass with viscid fluid content, highly echogenic density with shadowing, low level echoes and echogenic floaters located midline extends to the left adnexa measuring $10.9 \times 6.6 \times 9.9 \mathrm{~cm}$ (Figure $1 \mathrm{~b}$ ), with an impression of dermoid cyst. Past medical, family, social, sexual and menstrual history was unremarkable.

Abdominal examination revealed a palpable mass on the left lower quadrant area, cystic, movable and non-tender, measuring $12 \times 10 \mathrm{~cm}$. Internal examination revealed nulliparous introitus, smooth vaginal wall, cervix is midline, firm, uterus is unenlarged, the same abdominal mass is palpated at the left adnexal area measuring $12 \times 10 \times 8 \mathrm{~cm}$, cystic, fluctuant, movable, and slight tender on deep palpation. Digital rectal exam was unremarkable.

She underwent exploratory laparotomy. The left ovary was cystically enlarged to $10 \times 10 \times 8 \mathrm{~cm}$ with intact, thick whitish smooth capsule and areas with normal ovarian tissues (Figure 1c). The uterus, right ovary, bilateral fallopian tubes and appendix were grossly normal. Intraoperative diagnosis was dermoid cyst hence, left oophorocystectomy was done. Cut section showed a multilocular mass with cysts filled with brown turbid, sebaceous material and hair strands. Likewise seen was a solid area with fibrous to fatty cut surfaces.

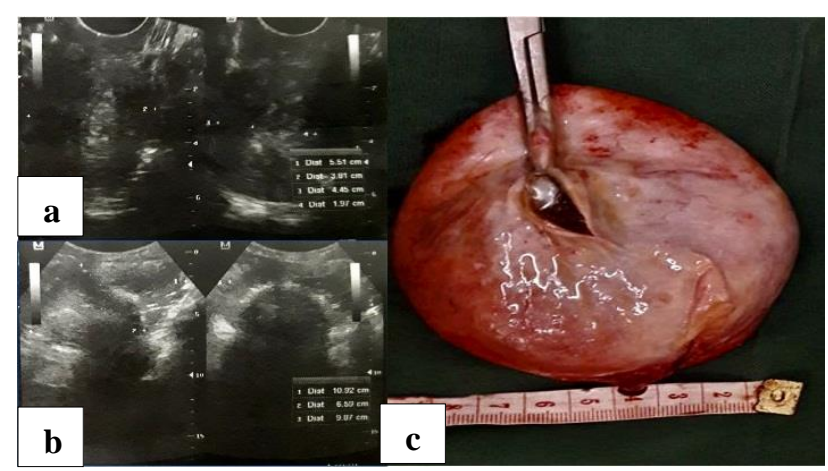

Figure 1: Ultrasound and Gross Morphology.

Microscopic scanning showed the portion of the left ovary lined by simple columnar epithelium with apical mucin and goblet cells. Noted underneath were nests of tumor cells with uniform and bland looking nuclei and pseudo stratified columnar epithelium with cilia (Figure 2a). Scans of the left ovary showed portion of inner surface lined by stratified squamous keratinizing epithelium. Likewise seen on the wall were sebaceous glands, transected hair shafts and fibrous tissues interspersed were neutrophils, lymphocytes, plasma cells and foamy macrophages and foreign -body giant cells (Figure 2b). There were nests of monotonous cells having small round to oval and course nuclei with scant cytoplasm (Figure 2c). These findings were suggestive of carcinoid tumor. Immunohistochemical staining showed positivity for synaptophysin (Figure 2d), and chromogranin (Figure 2e). Based on the morphological and immunohistochemical findings, the tumor was diagnosed as a carcinoid tumor arising from a mature cystic teratoma. No recurrence of the tumor for 30 months.

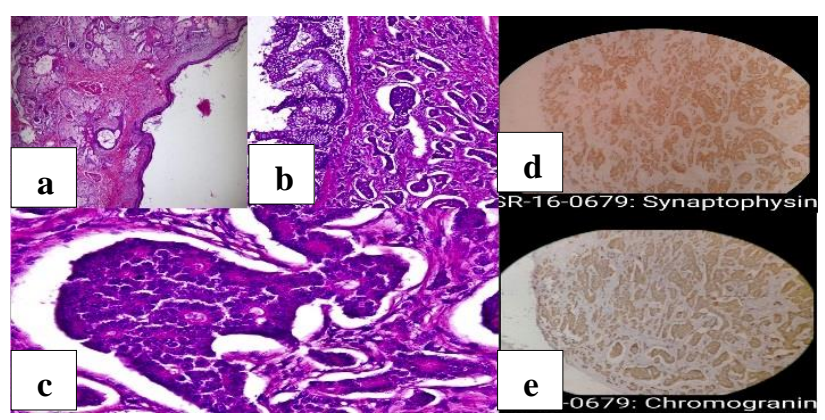

Figure 2: H\&E and Immunohistochemical Staining.

\section{Case 2}

A 52 year-old, female, G5P4 (4014) who presented with a 1-year history of gradually enlarging mass at the hypogastric area. Her past medical, family, social and sexual, histories were non-contributory. She had five pregnancies, the 2 nd had an abortive outcome and the rest were all delivered by normal spontaneous delivery and uncomplicated. She had her menopause at age of 49.

Eight months later, the mass gradually enlarged to approximately $10 \times 10 \mathrm{~cm}$ associated with pelvic heaviness. Abdominal ultrasound showed a pelvoabdominal mass, cystic, with septations, midline, and posterior to the uterus measuring $10.92 \times 8.82 \times 9.28 \mathrm{~cm}$ (Figure 3a). CA-125 value at $41.72 \mathrm{mIU} / \mathrm{ml}$.

Abdominal examination revealed a flabby, soft and nontender abdomen with a $10 \times 10 \mathrm{~cm}$, cystic, movable and non-tender mass. Pelvic examination revealed a normal external genitalia, parous introitus, smooth vaginal walls, cervix measures $2 \times 2 \mathrm{~cm}$, closed, firm and smooth, the uterus was small, with a $10 \times 10 \mathrm{~cm}$ left adnexal mass, cystic, movable and non-tender. The impression was an ovarian new growth, left probably malignant.

She underwent exploratory laparotomy. Intraoperatively, the left ovary was converted to a $12 \times 12 \times 12 \mathrm{~cm}$ cystic mass, with smooth and intact capsule. On cut section, the mass was multiloculated, multiseptated and contained brownish mucinous fluid. There were solid areas noted at the internal capsule with hemorrhages and necrosis (Figure 3b). A total hysterectomy with bilateral salpingooophorectomy, peritoneal fluid cytology infracolic omentectomy and pelvic lymph node dissection were done. The rest of the pelvic and abdominal organs were unremarkable (Figure 3c). Harvested lymph nodes were not suspicious for malignancy. Microscopic examination of the left ovary revealed a fibrous cyst wall with varisized colloid filled follicles resembling that of the thyroid (Figure $4 \mathrm{a}$ and b). Observed are small solid areas composed of compact microfollicles and trabecular 
patterns of follicular cells (Figure 4c and d) with enlarged, round, ovoid to pleomorphic, hyperchromatic to vesicular nuclei and some with prominent nucleoli and with interspersed mitoses (Figure 4e and f). Its wall was focally lined by respiratory type epithelium with underlying areas showing smooth muscle bundles (Figure 5a and b).

Immunohistochemistry demonstrated a positive result for Hector Battifora Mesothelial- 1 (HBME-1) in areas with tumor cells having nuclear grooves and nuclear inclusions (Figure 5c-f). Histopathologic evaluation of the left ovary demonstrated a follicular variant papillary thyroid carcinoma in a mature cystic teratoma, with metastasis to the right ovary. Thyroid gland evaluation was done to eliminate primary thyroid pathology. Serum thyroid function tests were within normal levels and the thyroid ultrasonography revealed a thyroid parenchymal disease with benign looking solid nodule in both lobes (Figure 3d). She refused to undergo any further management. For 24 months of follow up, she had normal pelvic examination as well as tumor markers and abdominal imaging results.
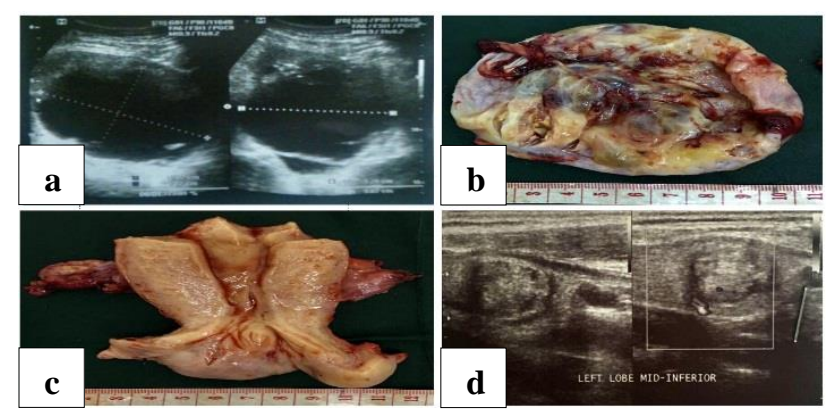

Figure 3: Ultrasound and Gross Morphology.

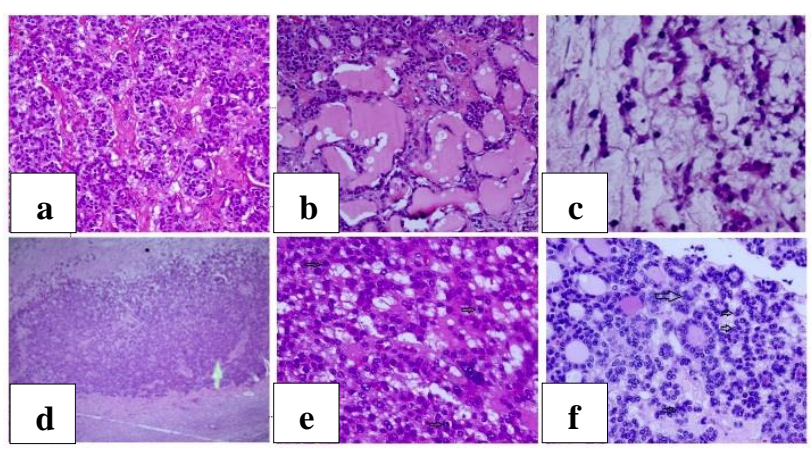

Figure 4: H\&E Staining.

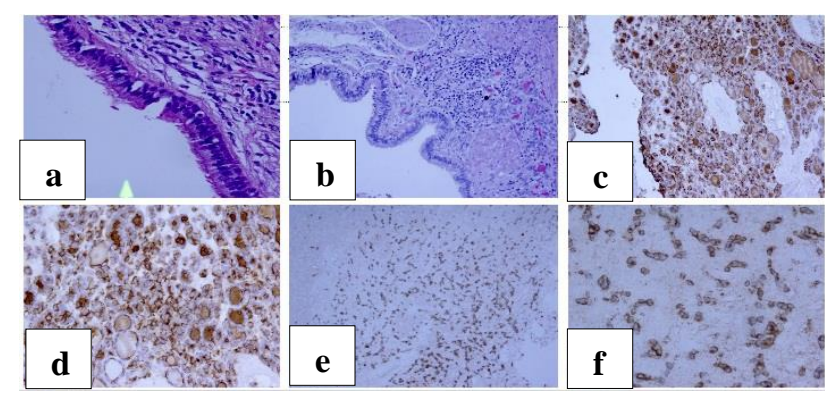

Figure 5: H\&E and Immunohistochemical Staining.

\section{DISCUSSION}

\section{Malignant transformation from MCTO}

Ovarian teratomas encompasses a number of histologic forms of tumors, all of which include mature or immature tissues of germ cell origin. The most frequent germ cell tumor is the benign cystic teratoma which consist of at least 2 tissue that recapitulates three layers of the developing embryo which includes ectoderm, mesoderm and endoderm. ${ }^{4}$ Benign teratoma accounts for more than $90 \%$ of germ cell tumors. Monodermal teratomas constitute primarily or solely of one tissue type. Ovarian monodermal tumors have three principal types: struma ovarii, carcinoid tumors, and lesions with neural differentiation. In its pure form, mature cystic teratoma is always benign, however malignant transformation has been reported, accounting for about $0.17-2 \%$. SCCA is the most common malignancy arising from MCT, accounting for $75 \%$, while other malignancies, such as adenocarcinoma, carcinoid tumor, sarcoma and papillary carcinoma are extremely rare with an estimate of 0.1 to $0.3 \% .^{2,3}$

The development of malignant transformation in MCT has not yet been expounded. A long term presence of MCT without removal is suggested to be associated with malignant transformation. Park et al reported that risk factors for malignant transformation include elevated CA125 levels, age over 40 years old, postmenopausal status and tumor size, in some studies the cut off size ranges from $10-11 \mathrm{~cm} .{ }^{5}$ The long term presence and relatively large size of the MCTO were the risk factors present on both cases. Other risk factors were all present on the latter.

Malignant change is rarely recognized preoperatively and no specific symptoms exist that would lead one to suspect malignant transformation developing from a MCTO. ${ }^{5}$ MCT can be easily diagnosed by imaging modalities and are usually easy to recognize on ultrasound. Since patients do not show with definite symptoms or signs that discriminates them from other diseases, or with specific radiologic finding, it is challenging to diagnose these sophisticated ovarian tumors and to establish the appropriate type of surgery prior to the operation. Medical conditions associated with dermoid cyst are thyrotoxicosis, autoimmune hemolytic anemia and carcinoid syndrome. The index patients did not present with any of these medical conditions.

The gross pathologic appearance of MCT is characteristic. However for a concealed malignancy, the following features are seen on MCT such as nodularity, papillary or cauliflower-like growths protruding into the cyst cavities or nodules or plaques within the walls, adhesions to the pelvic wall, peritoneum, and with the presence of ascites, areas of necrosis and hemorrhages, together with the presence of risk factors would aid physician to suspect malignancy. ${ }^{5}$ Solid growth and areas of necrosis and hemorrhages were apparent on both cases. 


\section{Carcinoid tumor}

Ovarian carcinoid tumors are monodermal teratomas occurring in a pure form $(15 \%)$ or combined with other teratomatous components (85\%), such as a dermoid cyst or a struma ovarii. ${ }^{6}$ Primary carcinoid tumors of the ovary are divided into strumal, trabecular, insular, and mucinous carcinoids. Strumal carcinoid is illustrated by the coexistence of carcinoid and thyroid tissue. Trabecular carcinoid is considered to be derived from hindgut or foregut, displays wavy ribbons or a trabecular arrangement of cells in a dense fibrous stroma. Insular carcinoid, the most popular type of ovarian carcinoid tumor is believed to be derived from midgut. The least common type of ovarian carcinoid is mucinous carcinoid which resembles a goblet cell carcinoid arising in the appendix. In this report, nests of monotonous cells having small round to oval and course nuclei with scant cytoplasm was seen consistent with insular carcinoid.

Carcinoid tumors are reactive to neuroendocrine markers, such as chromogranin, synaptophysin, and CD56. Chromogranin and synaptophysin are excellent discriminatory neuroendocrine markers for a carcinoid tumor. $^{6}$ In our case, immunohistochemical staining showed positivity to both chromogranin and synaptophysin.

Little data exists regarding the pathogenesis of primary carcinoid tumor of the ovary. However, recent studies suggest that molecular pathogenesis such as patient with inherited multiple endocrine neoplasia type 1 (MEN-1) are associated with carcinoid tumor. The p53 gene is only rarely mutated in carcinoid tumors. ${ }^{7}$ Risk factors and family history of the patient on hereditary malignancies are unremarkable.

\section{Papillary thyroid carcinoma}

Malignant transformation of thyroid tissue in MCTO can be classified histopathologically into three types. The most common type is papillary thyroid carcinoma (PTC) which is about $44 \%$, followed by follicular thyroid carcinoma (FTC) accounting for approximately $30 \%$. A unique type is the follicular variant of a papillary carcinoma which accounts for $26 \%$ of malignant degeneration. This variant is unique because it shares similar nuclear cytological features of a papillary carcinoma, it has however, a follicular architecture. It is a teratoma with absent predominance of thyroid tissue and with histological features of follicular variant papillary thyroid carcinoma. Thus, it is considered a papillary carcinoma on thyroid tissue in an ovarian teratoma. ${ }^{2,3}$

The application of immunohistochemical biomarkers may play an active or complementary role in the accurate classification. $^{8}$ HBME-1 is used as an immunohistochemistry stain to demonstrate that the thyroid tissue from the ovary is malignant nature of the ovarian thyroid component. In normal thyroid tissue, there is virtually no expression of HBME-1. Variety of biomarkers are reported, such as HBME-1, galectin-3, cytokeratin 19, CITED1, and TPO are most promising. However, in our institution, HBME-1 is the only available biomarker. The nonexistence of a primary lesion in the thyroid is necessary to disregard metastatic thyroid carcinoma to the ovary.

\section{Treatment}

The optimal management of these malignant transformations is uncertain because of its rarity and there is no gold standard and direct comparisons between treatment approach. The rarity suggests a low likelihood of future prospective studies to determine the best treatment options. $^{2}$ Furthermore, the treatment modalities for malignancy in mature cystic teratoma depend on the stage of the disease and fertility desire. Conservative treatment is often offered to younger patients who wish to retain their fertility and it consists of unilateral salpingooophorectomy or cystectomy without adjuvant therapy. Conservation of ovarian tissue on the affected side is acceptable. $^{2,9}$ For perimenopausal and postmenopausal women, bilateral salpingoophorectomy and hysterectomy should be performed. ${ }^{5}$ In postmenopausal women, complete cytoreduction surgery and lymphadenectomy, improves the treatment outcome. Unfavorable prognostic factors are mainly considered; cyst wall invasion, intraoperative rupture of the ovarian mass, tumor dissemination, and adhesions. Prognosis is excellent. There is no standardized approach to post treatment surveillance however routine physical examination and radiographic imaging are warranted.

The benefits of adjuvant radiotherapy or chemotherapy according to a retrospective study of Gainford et al have never been prospectively evaluated and remain unclear. ${ }^{10}$ In a surgical stage 1 tumors, further therapy is questionable given their relatively good prognosis. For patients with more advanced disease, there are no universally accepted regimens or dosage use. However, there has been a small number of case reports of prolonged survival in patients with a more advanced disease that have been treated with cisplatin-based chemotherapy.

\section{Prognosis and surveillance}

Malignant tumors arising from ovarian teratomas can occasionally present with locally invasive disease or with distant metastases. ${ }^{2}$ The predominant sites of metastasis are adjacent pelvic structures, including the contralateral ovary and hematologic dissemination may occur in the lungs, bone, liver and brain, however, metastasis is rare. In the first case, the lesion is localized in the removed ovary while the second case had a small focus of metastasis on the contralateral ovary as evident by a positive HBME-1, allotting a final stage IB.

Prognosis is dependent on the stage of the disease, with stage I patients having a relatively good prognosis, but the 
outcome is very poor when the disease has spread beyond the ovary. ${ }^{10}$ Other important predictors of prognosis include clinical stage, tumor grade, vascular involvement, and the mode of tumor infiltration.

In patients with lesions outside the pelvis like gastrointestinal or thyroid, it is recommended that a biopsy be made to rule out primary extraovarian malignancy. For the second case, a biopsy of the thyroid nodule was advised, however the patient refused. For these index cases, no adjuvant therapy was contemplated due to the early stages of disease. Both patients are in remission of the disease.

\section{CONCLUSION}

Malignant transformation in MCTO is rare yet diverse. Carcinoid tumor and papillary thyroid carcinoma are prototypes of extremely rare malignant transformation. The preoperative diagnosis of this complication is challenging, and the definitive diagnosis should be executed postoperatively. Since there are no specific symptoms that would indicate malignant transformation developing from MCTO, factors such as larger tumor size, solid areas with hemorrhage and necrosis could be beneficial in anticipating malignant transformation. Histopathologic examination of multiple tissue sections and immunohistochemical staining aids in definitive diagnosis. Complete excision of the tumor for these rare malignant transformation is curative, rendering excellent prognosis.

Funding: No funding sources Conflict of interest: None declared

Ethical approval: Not required

\section{REFERENCES}

1. Kim JY. A carcinoid tumor arising from a mature cystic teratoma in a 25-year-old patient: a case study, World J Surg Oncol. 2016;14:120.

2. Dane C, Ekmez M, Karaca A, Dane B. Case report of Follicular Variant of Papillary thyroid carcinoma arising from a dermoid cyst: A rare malignancy in a young women and review of literature. Taiwanese $\mathbf{J}$ Obstet Gynecol. 2012;51:421-5.

3. Souaf I, El Fatemi H, Bennani A. Papillary Carcinoma Derived from Ovarian Mature Cystic Teratoma: A New Case Report and Literature Review. Case Rep Clin Med. 2017;3:197-202.

4. Outwater E, Siegelman E, Hunt J. Ovarian Teratoma: Tumor types and imaging characteristics. Radio Graphics. 2001;21:475-79.

5. Park CH, Jung MH, Ji YI. Risk Factors for malignant transformation of mature cystic teratoma. Obstet Gynecol Sci. 2015;58(6):475-80.

6. Chun Y. Neuroendocrine Tumors of the Female Reproductive Tract: A Literature Review. J Pathol Transl Med. 2015;49(6):450-61.

7. Kamillaris C, Stratakis C. Multiple Endocrine Neoplasia Type 1 (MEN1): An Update and the Significance of Early Genetic and Clinical Diagnosis. Front Endocrinol (Lausanne) 2019;10:339.

8. Liu H, Lin F. Application of Immunohistochemistry in Thyroid Pathology. Arch Pathol Lab Med. 2015;139(1):67-82.

9. Guney N, Sayilgan T, Derin D, Ozcan D. Primary carcinoid tumor arising in a mature cystic teratoma of the ovary: a case report. Eur J Gynaecol Oncol. 2009;30(2):223-5.

10. Gainford MG, Tinker A, Carter J, Petru E, Nicklin J, et al. Malignant transformation within ovarian dermoid cysts: An audit of treatment reviewed and patient outcomes. An Australia New Zealand Gynecological Oncology Group (ANZGOG) and Gynecologic cancer Intergroup (CGIG) Study. Int J Gynecol Canc. 2010;20(1):75-81.

Cite this article as: Billod JA, Saure JG, Natavio KMA. Carcinoid tumor and papillary thyroid carcinoma arising from ovarian mature cystic teratoma: two cases of an extraordinary malignant transformation. Int J Reprod Contracept Obstet Gynecol 2021;10:1686-90. 